\title{
Inducible short-term and stable long-term cell culture systems reveal that the PAX3-FKHR fusion oncoprotein regulates CXCR4, PAX3, and PAX7 expression
}

\author{
Oana Tomescu ${ }^{1}$, Shujuan $\mathrm{J} \mathrm{Xia}^{1}$, Donna Strezlecki ${ }^{1}$, Jeannette L Bennicelli ${ }^{1, *}$, \\ Jill Ginsberg ${ }^{1, \dagger}$, Bruce Pawel ${ }^{2}$ and Frederic G Barr ${ }^{1}$ \\ ${ }^{1}$ Department of Pathology and Laboratory Medicine, University of Pennsylvania School of Medicine, \\ Philadelphia, PA, USA and ${ }^{2}$ Department of Pathology, Children's Hospital of Philadelphia, Philadelphia, \\ $P A, U S A$
}

\begin{abstract}
In the pediatric cancer alveolar rhabdomyosarcoma (ARMS), the 2;13 chromosomal translocation juxtaposes the PAX3 and FKHR genes to generate a chimeric transcription factor. To explore molecular pathways altered by this oncoprotein, we generated an inducible form by fusing PAX3-FKHR to a modified estrogen receptor ligand-binding domain and expressed this construct in the RD embryonal rhabdomyosarcoma cell line. This inducible system permits short-term evaluation of downstream expression targets of PAX3-FKHR and complements a panel of stable long-term RD subclones constitutively expressing PAX3-FKHR. Using these two sets of resources, we investigated several candidate PAX3-FKHR target genes. First, we demonstrated in both short-term and long-term systems that PAX3-FKHR upregulates expression of the gene encoding the chemokine receptor CXCR4. In addition, we found that expression of wild-type PAX3 is upregulated, whereas expression of wild-type PAX7 is downregulated by PAX3-FKHR. In the presence of cycloheximide, CXCR4 and PAX3 are still inducible, supporting the hypothesis that these genes are direct transcriptional targets of PAX3FKHR. Finally, studies of ARMS tumors revealed CXCR4, PAX3, and PAX7 expression levels consistent with our cell culture results. These findings of genes regulated by PAX3-FKHR will direct future biological and clinical investigation to important pathways contributing to ARMS tumorigenesis and progression.
\end{abstract}

Laboratory Investigation (2004) 84, 1060-1070, advance online publication, 7 June 2004; doi:10.1038/labinvest.3700125

Keywords: rhabdomyosarcoma; chromosomal translocation; gene fusion; transcription factor; gene expression

Alveolar rhabdomyosarcoma (ARMS) is an aggressive pediatric malignancy related to the myogenic lineage that is consistently associated with the $2 ; 13$ or 1;13 chromosomal translocation. ${ }^{1}$ These genetic events juxtapose the FKHR gene with either the $P A X 3$ or $P A X 7$ gene $^{2,3}$ and lead to changes in expression, function, and subcellular localization

Correspondence: Dr FG Barr, MD, PhD, Department of Pathology and Laboratory Medicine, University of Pennsylvania School of Medicine, 505C Stellar Chance Laboratories, 422 Curie Boulevard, Philadelphia, PA 19104, USA.

E-mail: barrfg@mail.med.upenn.edu.

${ }^{*}$ Current address: Kirby Center for Molecular Ophthalmology, Scheie Eye Institute, 310 Stellar Chance Laboratories, 422 Curie Blvd, Philadelphia, PA 19104, USA.

${ }^{\dagger}$ Current address: Division of Oncology, Children's Hospital of Philadelphia, 34th and Civic Center Boulevard, Philadelphia, PA 19104, USA.

Received 10 November 2003; revised and accepted 18 March 2004; published online 7 June 2004 of the associated gene products. First, the fusion genes express PAX3-FKHR and PAX7-FKHR fusion transcripts that are expressed at higher levels than the corresponding wild-type transcripts. ${ }^{4}$ Second, these fusion transcripts encode proteins in which the amino-terminal (N-terminal) PAX3 or PAX7 DNA-binding domain is joined with the carboxy-terminal (C-terminal) FKHR transactivation domain. In transcriptional assays, these fusions proteins are more potent activators than wild-type PAX3 or PAX7. ${ }^{5,6}$ Third, the PAX3-FKHR fusion protein localizes exclusively to the nucleus and is thus constitutively active in contrast to the regulated localization of the wild-type FKHR protein. ${ }^{7}$ Therefore, these combined effects result in high levels of constitutively active nuclear chimeric transcription factors that excessively activate transcription of genes with PAX3/PAX7 DNA-binding sites. 
Previous cell culture studies identified several phenotypic consequences of PAX3-FKHR consistent with oncogenic function. Introduction of PAX3FKHR expression constructs into susceptible cells leads to oncogenic transformation. ${ }^{8,9}$ In addition, inhibition of PAX3-FKHR expression or function impairs survival of ARMS tumor cells. ${ }^{10,11}$ Finally, other studies suggest that transfer of the PAX3FKHR cDNA commits cells to the myogenic lineage, but maintains them in a proliferative terminally undifferentiated state. ${ }^{12,13}$

The search for downstream targets of PAX3-FKHR seeks to provide additional insights into the cellular and molecular pathways that are disturbed during ARMS tumorigenesis. We previously described initial gene transfer studies of myogenic cell culture systems that were utilized to evaluate putative downstream targets of PAX3-FKHR. ${ }^{14}$ In particular, we generated stable PAX3-FKHR-expressing subclones of the RD embryonal rhabdomyosarcoma (ERMS) cell line to evaluate long-term gene expression alterations in the presence of PAX3-FKHR. Using this system, we were able to demonstrate that the MET gene, which encodes the HGF/SF receptor involved in growth and motility pathways, is a downstream target of PAX3-FKHR. In the current study, we extended our target gene analysis by examining the immediate consequences of forced PAX3-FKHR expression. Towards this end, we developed an inducible PAX3-FKHR-estrogen receptor (ER) ligand-binding domain (P3FK/ER) construct that is functionally dependent on the presence of 4-hydroxytamoxifen (4-OHT). We use this inducible system as well as the stable subclones to demonstrate that PAX3-FKHR can modulate the expression of putative target genes CXCR4, PAX3, and $P A X 7$ in both short-term and long-term settings. Our findings indicate that there are subsets of PAX3FKHR target genes whose expression is deregulated by PAX3-FKHR through distinct mechanisms, and provide insight into the cellular pathways that are deregulated during the progression of ARMS tumors.

\section{Materials and methods}

\section{Tumor Cell Lines and Specimens}

Maintenance of ERMS cell line RD and ARMS cell lines RH5, RH18, MP-4, TTC280, RH30, and RH28 was performed as previously described., ${ }^{4,15}$ Tumor specimens were collected and screened by reverse transcription (RT)-polymerase chain reaction (PCR) for expression of the PAX3-FKHR and PAX7-FKHR fusion transcripts as described previously. ${ }^{16}$

\section{Construction of Expression Plasmids}

The plasmid pcD3M8 contains the complete PAX3-FKHR open reading frame in pcDNA3
(Invitrogen). ${ }^{5}$ A plasmid containing the modified ER ligand-binding domain cDNA was provided by T Littlewood. ${ }^{17}$ The ER ligand-binding domain was amplified with primers containing a SacII site, $5^{\prime}$-TCCCCGCGGGGATCCACGAAATGAAATG- $3^{\prime}$ and $5^{\prime}$-TAACCGCGGCACTAGTAGGAGCTCTCAGA-3'. A SacII site (underlined) was introduced into the PAX3-FKHR stop codon in pcD3M8 by in vitro mutagenesis using the QuikChange Site-Directed Mutagenesis Kit (Stratagene) with the oligonucleotide 5'-CCTGCTCACTAACCCGCGGCCTGACACC CAGCT-3' and a complementary oligonucleotide. The ER ligand-binding domain PCR fragment was ligated into the mutagenized pcD3M8 plasmid digested with SacII to generate the P3FK/ERpcDNA3 vector.

The mscv2.2-derived retroviral vector pK1 (provided by $\mathrm{W}$ Pear) contains a multiple cloning site upstream of an internal ribosomal entry site and a gene for puromycin resistance. To clone P3FK/ER into pK1, a blunt-ended SacI fragment of P3FK/ER was excised from pcDNA3 and cloned into HpaIlinearized pK1. PAX3-FKHR-pK1 was cloned by ligation of the XhoI/NotI fragment of PAX3-FKHR into XhoI/NotI digested pK1.

\section{Retroviral Transduction of RD Cells}

Transgene-expressing pK1 constructs were co-transfected into 293T cells with the packaging vector pCL-10A1 (RetroMax, Imgenex). Supernatants containing retroviral particles were harvested after $48 \mathrm{~h}$ and used to infect target cells. Transduced cells were selected for 3 days in medium supplemented with $1 \mu \mathrm{g} / \mathrm{ml}$ puromycin. Short-term populations were expanded, with 4-OHT induction as indicated, and then RNA was isolated for analysis in ribonuclease (RNase) protection experiments. Stable PAX3-FKHR subclones were generated by seeding selected cells in puromycin-supplemented medium $(1 \mu \mathrm{g} / \mathrm{ml})$ for 2 weeks.

\section{Transient Transfections and Luciferase Reporter Assays}

To generate the reporter plasmid 6xPRS-9 TATALucS, the SalI/BamHI fragment of 6xPRS-9TKCAT (provided by P Gruss) ${ }^{18}$ was inserted into BamHI/ SalI fragment of LucS (provided by M Busslinger). Luciferase reporter transcription experiments were performed by calcium phosphate-mediated transient transfection of RD-pK1 or RD-P3FK/ER cells (seeded at $10^{4} / \mathrm{cm}^{2}$ in 24 -well plates). Cells were transfected with $170 \mathrm{ng}$ reporter (LucS or 6xPRS-9 LucS). Lysates of transfected cells were assayed for expression of firefly luciferase reporter gene and the control renilla luciferase gene using a dual luciferase assay kit (Promega). The amounts of cell lysates tested were normalized for transfection efficiency, as determined by expression of renilla luciferase from 
17 ng pRLTK cotransfected plasmid. Results are expressed as absolute luciferase units.

\section{CHX Experiments}

For CHX experiments, RD-P3FK/ER and RD-pK1 control cells were expanded and treated with $10 \mu \mathrm{M}$ CHX for $1 \mathrm{~h}$ prior to $24 \mathrm{~h}$ induction with the indicated level of 4-OHT (0 or $100 \mathrm{nM}$ ).

\section{Riboprobe Plasmids}

To detect P3FK/ER expression, a P3FK/ER-specific riboprobe plasmid was assembled by cloning a blunt-ended HindIII/NcoI fragment (405 bp) corresponding to the C-terminal portion of FKHR and the first $360 \mathrm{bp}$ of ER ligand-binding domain sequence into pSP72 (Promega). A CXCR4-specific riboprobe plasmid was assembled by cloning a blunt-ended EcoRI/HincII fragment (400 bp) of CXCR4 cDNA (provided by R Doms) corresponding to exons 1-2 into PvuII-linearized pSP72. PAX3-FKHR, PAX3 and PAX7 riboprobes were previously described. ${ }^{4}$ A glyceraldehyde-3-phosphate dehydrogenase (GAPDH) riboprobe plasmid (pTRI-GAPDH, Ambion) served as an internal control for all RNase protection assays.

\section{RNase Protection Analysis}

Total RNA was isolated from cells as described. ${ }^{16}$ An aliquot of total cellular RNA ( $10 \mu \mathrm{g})$ was hybridized with both [ $\left.{ }^{32} \mathrm{P}\right] \mathrm{UTP}$-labeled test and GAPDH control antisense probes and digested with A/T1 RNases (RPAII Kit; Ambion). Protected bands were quantified by phosphorimaging (Molecular Dynamics) and normalized for the uridine content of each riboprobe. The ratio of test RNA to GAPDH RNA was calculated for each sample. Additionally, to standardize these experiments and allow for comparison among different RNA samples, all test/GAPDH ratios were normalized to PAX3 transcript levels (arbitrarily set to 10 expression units) determined for the ERMS cell line RD, which also served as a control for RNase protection assay integrity. All RNase protection results are thus graphically depicted as 'relative units' (RU) of transcript expression. Control experiments demonstrate that GAPDH expression does not change in the presence of PAX3FKHR and that the variation between experiments and among identical samples within one experiment was less than $15 \%$ (data not shown). Relative expression levels below $2 \mathrm{U}$ are at the level of threshold for this assay.

\section{Quantitative RT-PCR}

CXCR4 mRNA levels were measured by quantitative RT-PCR assays performed using TaqMan methodo- logy on an ABI Prism 7700 Sequence Detection System. In these RT-PCR assays, the CXCR4-specific PCR primers were GAACCAGCGGTTACCATGGA and GGGTTCCTTCATGGAGTCATAGTC and the fluorescent probe was TATATACACTTCAGATAAC TACACCGAGGAAATGGGCTTAMRA linked to 6FAM. As an internal control, 18S RNA was monitored in a multiplexed reaction with commercially available primers and probes (ABI). Primer concentrations for $18 \mathrm{~S}$ and CXCR4 were adjusted to 0.05 and $0.1 \mathrm{mM}$, respectively.

\section{Results}

\section{Expression of PAX3-FKHR in a Myogenic and Neoplastic Background}

To study gene expression changes mediated by the PAX3-FKHR transcription factor, we expressed PAX3-FKHR in the fusion-negative myogenic ERMS cell line RD. To examine long-term consequences of PAX3-FKHR expression, we expanded our previous series of stable $\mathrm{RD}$ subclones engineered to express a constitutively active PAX3-FKHR construct and detected transcript expression in the range of 16-200 RU (Figure 1a). PAX3-FKHR transcript expression was undetectable in $31.6 \%(14 / 45)$ of the selected subclones, and was detected from 1 to $50 \mathrm{RU}$ in $28.9 \%$ $(13 / 45)$ and from 51 to $100 \mathrm{RU}$ in $26.7 \%(12 / 45)$ of subclones isolated. Higher levels of PAX3FKHR expression, detected from 101 to 150 RU and 151 to 200 RU were evident in $6.7 \%(3 / 45)$ of the subclones in each category. A series of ARMS cell lines demonstrated comparable levels of PAX3-FKHR expression (47-185 RU) with a similar distribution (Figure 1a). Control pK1-transduced subclones were isolated and served as negative controls in our downstream target gene analyses (data not shown).

To examine the immediate consequences of PAX3FKHR expression, we generated an inducible PAX3-FKHR construct consisting of PAX3-FKHR sequences fused to the modified ER ligand-binding domain ${ }^{17}$ resulting in a 4 -OHT-inducible protein P3FK/ER. Transduction of RD cells with this P3FK/ER construct resulted in a population of cells that expressed the P3FK/ER transcript at levels comparable to endogenous PAX3-FKHR transcript expression in several ARMS cell lines (data not shown). To determine whether the P3FK/ER protein demonstrated 4-OHT-inducible transcriptional function, RD-P3FK/ER cells were transiently transfected with a luciferase reporter gene construct containing multimerized PAX3 (6xPRS-9) DNA-binding sites and were treated with varying concentrations of 4-OHT. Inducible PAX3-FKHR function was confirmed by the finding of 4-OHTdependent transactivation (Figure 1b). Maximal reporter gene transactivation occurred with 50$100 \mathrm{nM}$ 4-OHT; a 37.5-fold difference in luciferase 

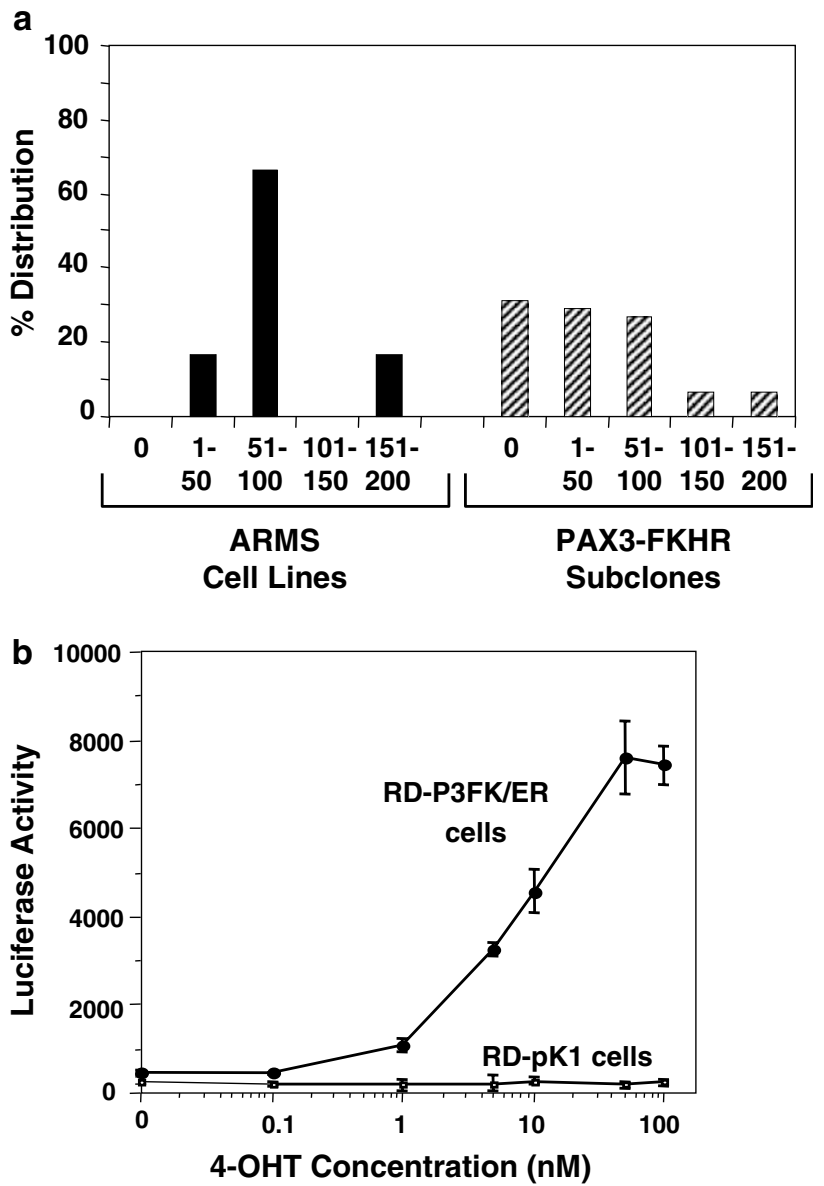

Figure 1 PAX3-FKHR expression in stable constitutively expressing subclones and transcriptional activity in inducible system. (a) Distribution of PAX3-FKHR expression in ARMS cell lines and RD stable subclones. Stable PAX3-FKHR subclones were generated by seeding transduced RD cells in puromycin-supplemented medium $(1 \mu \mathrm{g} / \mathrm{ml})$ for 2 weeks. Additional PAX3-FKHR subclones were obtained by calcium phosphate transfection and selection in G418-supplemented medium $(1 \mathrm{mg} / \mathrm{ml})$. Levels of exogenous PAX3-FKHR transcript expression were quantified by RNase protection analysis and compared to the levels of endogenous PAX3-FKHR expression in six ARMS cell lines. (b) Dose-response curve of transcriptional activity in inducible RD-P3FK/ER cells. $\mathrm{RD}$ cells transduced with P3FK/ER or control pK1 constructs were selected in puromycin-supplemented medium for 3 days and then transiently transfected with a luciferase gene reporter construct containing multimerized PAX3 binding sites. Luciferase activity was measured in cell lysates of RD-pK1 and RD-P3FK/ER treated with $4-\mathrm{OHT}(0-100 \mathrm{nM})$.

gene transactivation between RD-P3FK/ER cells and RD-pK1 cells was found at $100 \mathrm{nM}$ 4-OHT. In the absence of induction, there was a 1.6-fold difference in luciferase gene transactivation between RD-P3FK/ER cells (461 $\pm 50 \mathrm{U})$ and RD-pK1 cells $(282 \pm 73 \mathrm{U})$, indicating that the P3FK/ER fusion is slightly active even in the absence of inducing agent. Neither RD-pK1 nor RD-P3FK/ER cells demonstrated significant expression of a luciferase reporter that did not contain 6xPRS-9 binding sites, regardless of 4-OHT-concentration (data not shown).

\section{Growth Effects of PAX3-FKHR in RD Cells}

In our previous study of subclones of RD cells expressing varying levels of PAX3-FKHR, we investigated whether the presence of the fusion oncoprotein changed the growth properties of the tumor cells. ${ }^{14}$ In particular, we assayed focus formation and serum dependence and found no enhancement associated with PAX3-FKHR expression. More recently, another group investigating PAX3-FKHR-expressing subclones of ERMS cell lines reported enhanced cellular proliferation under low serum growth conditions. ${ }^{19}$ As we observed significant heterogeneity in growth properties among the various PAX3-FKHR-expressing subclones in our stable constitutively expressing subclones (data not shown), we chose to further address these issues with an early culture of RD-P3FK/ERtransduced cells and a comparable control culture transduced with pK1 vector. Each cell type was plated in either serum-free or serum-containing $(10 \%$ FBS $)$ medium with or without 5,10 , or $100 \mathrm{nM}$ 4-OHT. As demonstrated in Figure 2, the growth curve in cells expressing active PAX3-FKHR was depressed to varying degrees relative to the control culture and there was no evidence of increased serum independence. Therefore, these findings support the view that $\mathrm{RD}$ is a highly evolved tumor cell line whose cell culture growth properties are not directly stimulated by expression of the PAX3-FKHR oncoprotein. However, as shown below, RD cells provide a very useful human myogenic system for examining downstream targets of PAX3-FKHR action in a variety of cellular pathways.

\section{CXCR4 Gene Expression is Regulated by PAX3-FKHR}

CXCR4 encodes a G-protein coupled chemokine receptor whose ligand is CXCL12/stromal derived factor-1 (SDF-1). ${ }^{20}$ CXCR4/SDF-1 signaling induces cytoskeletal rearrangements, adhesion to endothelial cells, and directional migration across vascular barriers, which are mechanisms utilized by normal hematopoietic cells during migration/homing and potentially by tumor cells during metastasis. Recently, in a FACS analysis of CXCR4 cell surface expression, we demonstrated that there is greater expression in ARMS than in ERMS cell lines, and hypothesized that CXCR4 may be a downstream target gene of PAX3-FKHR. ${ }^{21}$ To test this hypothesis, we first evaluated whether CXCR4 expression is induced in subclones stably expressing PAX3-FKHR (Figure 3a). Subclones were selected to represent various expression levels of PAX3-FKHR. Control (c1-c2) and PAX3-FKHR nonexpressing subclones (n1-n2) displayed undetectable levels of CXCR4 transcript, while PAX3-FKHR-expressing RD subclones demonstrated upregulation of CXCR4 that correlated directly with the level of PAX3-FKHR 

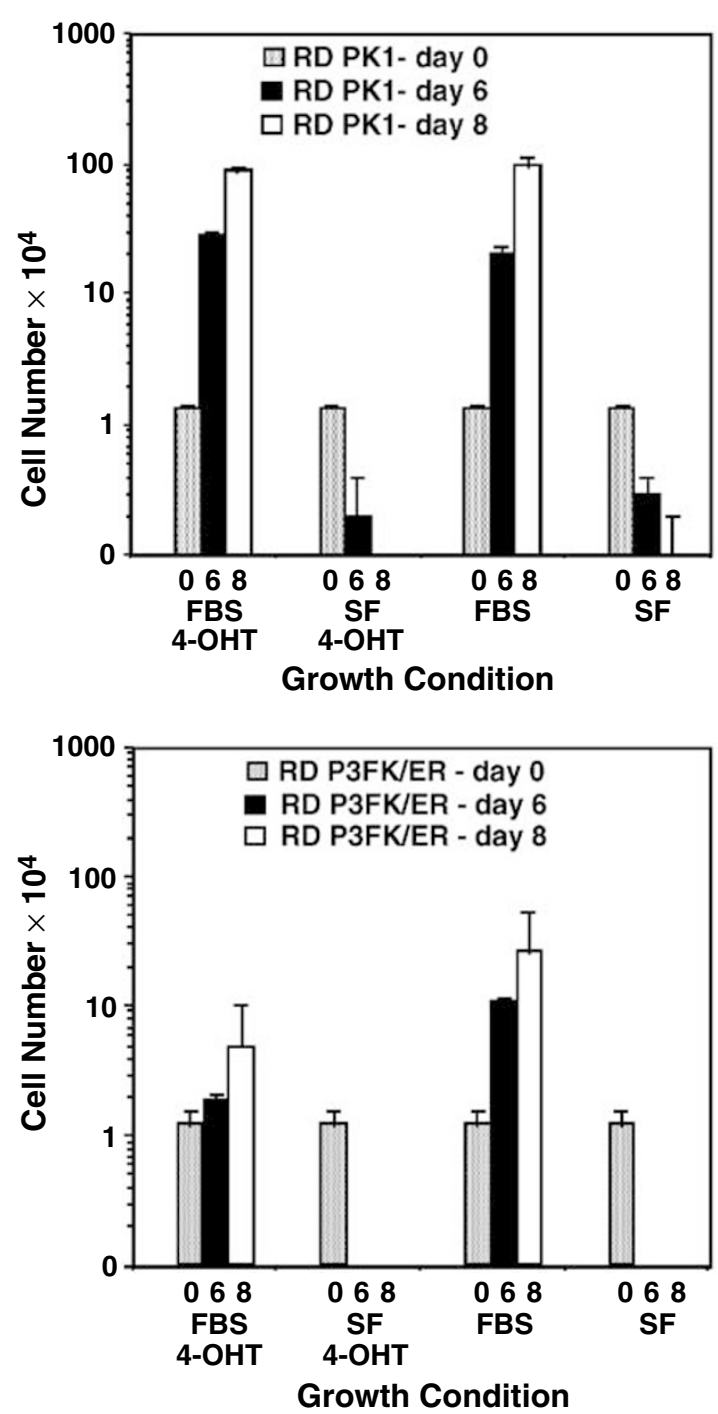

Figure 2 Growth of uninduced and induced P3FK/ER cells in high and low serum. RD cells transduced with control PK1 construct (left) or P3FK/ER (right) were plated in multiple well plates with DMEM medium supplemented with $10 \%$ fetal bovine serum (FBS) with or without $100 \mathrm{nM} 4-\mathrm{OHT}$. After $24 \mathrm{~h}$ (day 0), the medium was changed to either $10 \%$ FBS or $0 \%$ FBS (SF). At the indicated time points, three wells were trypsinized and the cells were counted manually with a hemocytometer. In addition, at days 2,4 , and 6 , the medium in the remaining wells was changed.

expression. Linear regression analysis of the series revealed a strong positive correlation $(r=0.944$; $P<0.0001 ; n=14$ ) between PAX3-FKHR and CXCR4 transcript levels.

We also evaluated short-term alterations in CXCR4 expression in inducible RD-P3FK/ER cells (Figure 3b). RD-P3FK/ER cells incubated with varying 4-OHT concentrations (0-100 nM) demonstrated 4-OHT-correlated increases in CXCR4 expression, whereas control RD-pK1 cells expressed undetectable CXCR4 mRNA levels, regardless of the 4-OHT concentration. Maximal CXCR4 expression, which measured $155 \mathrm{RU}$, was achieved with $100 \mathrm{nM}$
4-OHT-induction in the RD-P3FK/ER cells. It should also be noted that uninduced RD-P3FK/ER cells (0 nM 4-OHT) showed an increase in CXCR4 expression relative to pK1-transduced cells to 13 RU. This finding has been replicated in independent experiments (data not shown) and corroborates the results of the reporter assay (Figure 1b) that indicated a low level of P3FK/ER transcriptional activity in the absence of 4-OHT.

To provide evidence that CXCR4 induction may be a direct effect of PAX3-FKHR, we treated RD-pK1 and RD-P3FK/ER cells with the protein synthesis inhibitor CHX prior to induction with 4-OHT (Figure 3c). ${ }^{22}$ The ability of 4-OHT to induce CXCR4 expression in the presence of CHX supports the hypothesis that the CXCR4 gene is a direct transcriptional target of the PAX3-FKHR protein. In addition, the baseline level of CXCR4 expression was elevated in all CHX-treated cells (compare Figure $3 \mathrm{~b}$ to $\mathrm{c}$ ), suggesting the presence of a labile repressor that regulates CXCR4 expression in both RD-pK1 and RD-P3FK/ER cells and functions independently of PAX3-FKHR.

Finally, we developed a quantitative RT-PCR assay to measure CXCR4 mRNA levels in RMS tumors and thereby determine the potential significance of these findings in the in vivo setting. In 12 of 13 PAX3-FKHR-expressing ARMS tumors tested, there was readily detectable RNA expression that spanned over a 10-fold range (Figure 4). However, further analyses of PAX7-FKHR-expressing ARMS tumors and fusion-negative ERMS tumors demonstrated comparable levels of CXCR4 expression as seen in the PAX3-FKHR-expressing ARMS tumors (Figure 4). Therefore, we surmise that CXCR4 is also a downstream target of PAX7-FKHR and that other transcriptional mechanisms must be involved, perhaps involving wild-type PAX3 or PAX7, to upregulate expression of CXCR4 in ERMS cells. ${ }^{23}$

\section{PAX3-FKHR Differentially Regulates the PAX3/PAX7 Subfamily of Genes}

We have previously shown that the PAX3-FKHR and $P A X 7-F K H R$ fusion genes are overexpressed in ARMS tumors relative to their wild-type counterparts, $P A X 3$ and $P A X 7.4$ We hypothesized that the wild-type PAX3 and PAX7 proteins compete with the fusion oncoproteins for DNA-binding sites and/ or protein interactions and that in order to circumvent such interference, the PAX3-FKHR and PAX7FKHR oncoproteins may downregulate wild-type $P A X 3$ or $P A X 7$ gene expression. To address this hypothesis, we investigated whether there are alterations in endogenous wild-type PAX3 and PAX7 expression in the long-term presence of PAX3-FKHR (Figure 5a). Surprisingly, mean PAX3 expression was upregulated 2.6-fold in the complete series of PAX3-FKHR-expressing subclones as compared to control vector-transduced subclones 


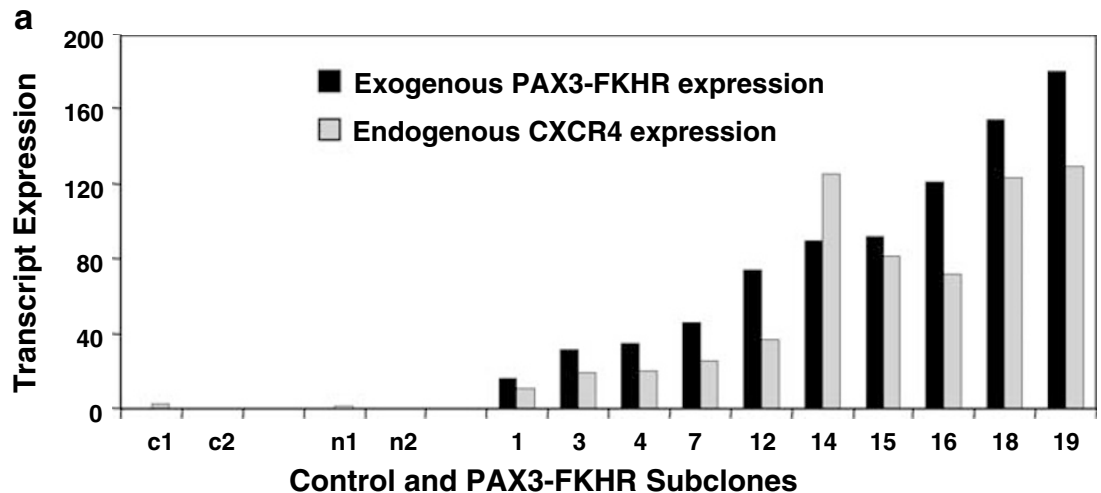

b

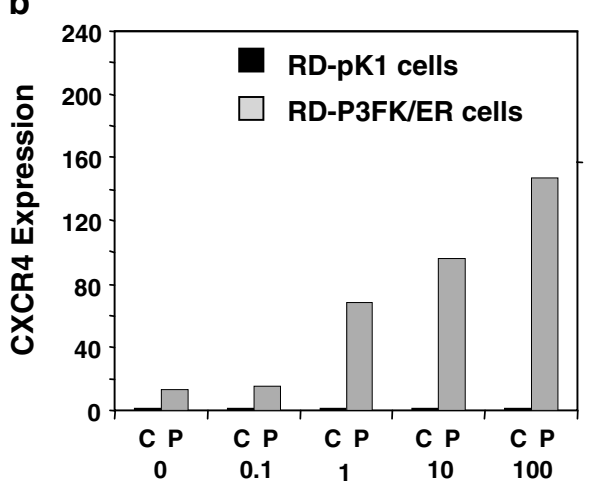

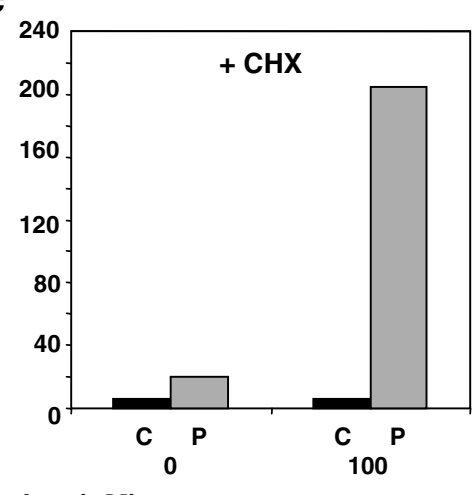

Figure 3 CXCR4 mRNA expression in response to exogenous PAX3-FKHR in RD ERMS cells. (a) CXCR4 expression in stable PAX3-FKHR expressing subclones. CXCR4 (gray bars) and PAX3-FKHR (black bars) mRNA expression was quantified in total RNA isolated from pK1 control (c1-c2), PAX3-FKHR nonexpressing (n1-n2) and a series of PAX3-FKHR-expressing RD subclones by RNase protection analysis. (b) Dose-dependent induction of CXCR4 expression in RD-P3FK/ER cells by 4-OHT. RD-pK1 (C/black bars) or RD-P3FK/ER (P/gray bars) cells were incubated with a range of $4-\mathrm{OHT}$ concentrations $(0-100 \mathrm{nM})$ for $24 \mathrm{~h}$, total RNA was isolated, and CXCR4 expression was quantified by RNase protection analysis. Although no error bars are shown, results have been corroborated in independent experiments. (c) CXCR4 expression in CHX-treated RD-P3FK/ER and RD-pK1 cells. RD-pK1 (C/black bars) or RD-P3FK/ER (P/gray bars) cells were treated with $10 \mu \mathrm{M}$ CHX for $1 \mathrm{~h}$ prior to 24 -h induction with mock or $100 \mathrm{nM} 4-\mathrm{OHT}$. Total RNA was isolated and CXCR4 expression was measured by RNase protection analysis.

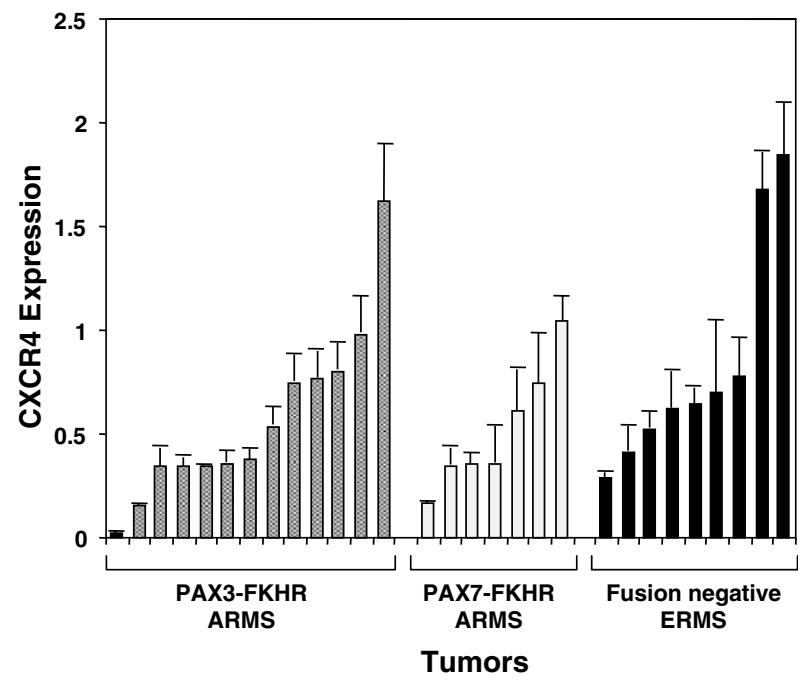

Figure 4 CXCR4 mRNA expression in ARMS and ERMS tumors. CXCR4 and 18S RNA transcript levels were measured in triplicate in each sample by a quantitative RT-PCR assay using TaqMan methodology. The relative levels were extrapolated from standard curves generated during the PCR run, and an average test to control ratio ( \pm s.d.) was calculated for each specimen.
$(25.8 \pm 7.6$ and $10.2 \pm 2.6 \mathrm{RU}, \quad$ respectively; $P<0.0001)$. In contrast, PAX7 expression was undetectable in almost all of PAX3-FKHR-expressing subclones; mean PAX7 expression was reduced by over 43-fold in the PAX3-FKHR-expressing subclones as compared to vector-transduced controls $(1.5 \pm 3.3$ and $65.3 \pm 22.7 \mathrm{RU}$, respectively; $P<0.0001)$. Similar results were obtained in a series of long-term stable PAX7-FKHR-expressing subclones (data not shown).

We next investigated whether upregulation of PAX3 expression was also evident in the presence of the inducible P3FK/ER construct (Figure 5b, left panel). In the absence of 4-OHT, PAX3 expression in RD-P3FK/ER cells (5.0 RU) was higher than the level in RD-pK1 cells (3.8 RU), in accord with the low basal activity of fusion protein seen in other experiments. To further confirm that wild-type $P A X 3$ is a downstream target of PAX3-FKHR, induction of RD-P3FK/ER cells with $100 \mathrm{nM} 4$ OHT resulted in upregulation of PAX3 expression to 13 RU. Furthermore, when we treated cells with the protein synthesis inhibitor CHX prior to 

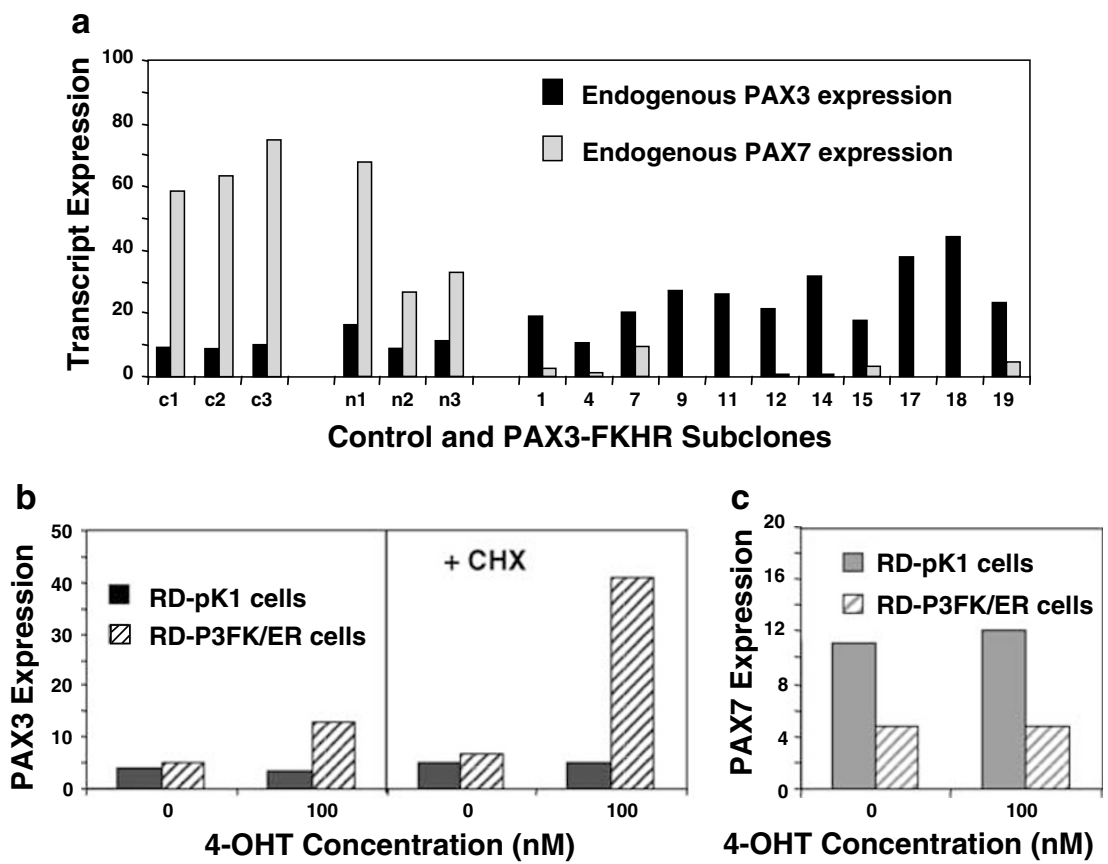

Figure 5 Differential regulation of PAX3 and PAX7 in response to exogenous PAX3-FKHR in RD cells. (a) PAX3 and PAX7 mRNA expression in stable PAX3-FKHR-expressing subclones. PAX3 (black bars) and PAX7 (gray bars) transcript expression was quantified in total RNA samples isolated from a series of control (c1-c3), nonexpressing (n1-n3) and PAX3-FKHR-expressing subclones by RNase protection analysis. PAX3-FKHR transcript levels are undetectable in subclones c1-c3 and n1-n3 and measured 16, 34, 46, 54, 67, 74, 90, $92,147,155$, and 180 RU in the other subclones in the order shown. The control and test subclones shown represent only a subset of the subclones isolated in the described experiments. (b) Induction of PAX3 expression in RD-P3FK/ER cells by 4-OHT. PAX3 transcript levels were measured in untreated (left panel) or CHX-treated (right panel) RD-P3FK/ER (hatched bars) or RD-pK1 (solid bars) cells. Cells were treated with $10 \mu \mathrm{M}$ CHX for $1 \mathrm{~h}$ prior to 24 -h induction with 4-OHT (mock or $100 \mathrm{nM}$ ) and harvested for RNA. PAX3 expression was then measured by RNase protection analysis. (c) PAX7 expression in the presence of P3FK/ER. PAX7 transcript levels were measured in RDP3FK/ER (hatched bars) or RD-pK1 (solid bars) cells. After cells were induced with $100 \mathrm{nM} 4-\mathrm{OHT}$ or mock treatment for $24 \mathrm{~h}$, RNA was isolated and the level of endogenous PAX7 was assayed by RNase protection analysis. It should be noted that because of the absence of a 4-OHT-induced effect, an experiment with CHX is not relevant in this situation.

induction with 4-OHT (Figure 5b, right panel), not only is PAX3 upregulated in the presence of CHX, but there is also a 8.2 -fold increase in PAX3 expression in CHX-treated RD-P3FK/ER cells (41 RU). This superinduction is consistent with the presence of a labile repressor that interacts with PAX3-FKHR. These findings thus suggest that the $P A X 3$ gene may be a direct downstream target of the fusion transcription factor and that additional proteins are involved in the regulation of this gene.

In contrast to upregulation of PAX3 expression by PAX3-FKHR, PAX7 expression was downregulated in the presence of P3FK/ER (Figure 5c). RD-pK1 cells express moderate levels of PAX7, which were not altered by treatment with 4-OHT (10.7 and 11.8 RU, respectively). In RD-P3FK/ER cells without 4-OHT treatment, there was 2.2-fold less PAX7 expression 4.8 RU) as compared to RD-pK1 cells without 4-OHT treatment. Furthermore, treatment of the RD-P3FK/ER cells with $100 \mathrm{nM} 4-\mathrm{OHT}$ does not result in any additional decreases in PAX7 mRNA levels, and thus it appears that the low level of PAX3-FKHR activity in the uninduced cells is sufficient to downregulate the PAX7 expression to this intermediate level. Therefore, the downregulation of PAX7 expression appears to be divisible into two phases. In one phase, which occurs at very low PAX3-FKHR activities, there is an initial decrease in PAX7 expression. The PAX3-FKHR level required for this effect is so low that that the basal level of PAX3-FKHR activity in the P3FK/ER inducible system (which is clearly greater than background) is sufficient to elicit this effect. The second phase of PAX7 downregulation is only seen in the long-term stable PAX3-FKHR subclones in which there is near complete extinction of PAX7 expression. Together the data suggest that PAX7 expression, which is completely extinguished in the stable PAX3-FKHR stable subclones but only modestly downregulated in the inducible model, is more sensitive to the longterm effects of PAX3-FKHR. These findings may indicate a requirement for replication either for additional levels of gene silencing (such as assembly of an inactive chromatin complex) or for actual selection against PAX7 expression.

To determine whether the differential regulation of wild-type PAX3 and PAX7 expression occurs within the same tumor, we measured the wild-type PAX3 and PAX7 mRNA levels in a series of PAX3FKHR-expressing ARMS tumors by RNase protection analysis (Figure 6). In that riboprobes were concurrently labeled and hybridized to near 


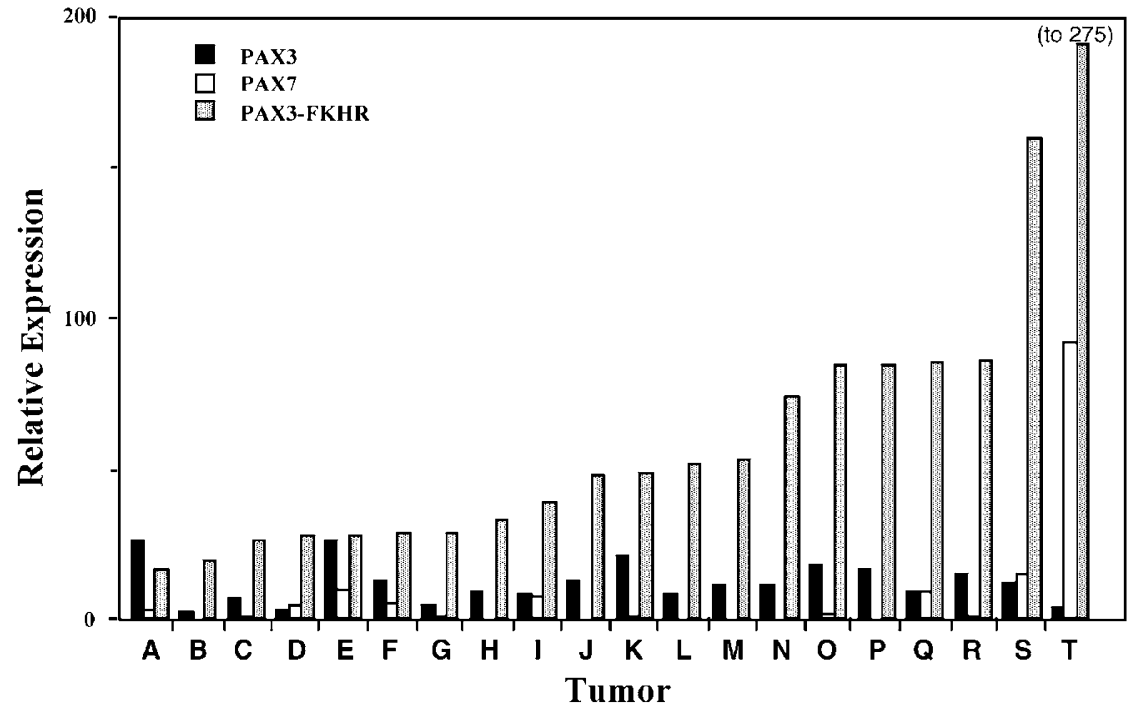

Figure 6 Relative expression of wild-type PAX3 and PAX7 in PAX3-FKHR-positive ARMS tumors. Total RNA from the indicated tumors was hybridized with [32P]UTP-labeled test (either PAX3, PAX7, or PAX3-FKHR) and control (GAPDH) riboprobes. RNase protection analysis was performed, the protected bands were quantified by phosphorimaging and normalized for the number of uridines in each antisense probe, and a test RNA/GAPDH RNA ratio was calculated. Results are shown as expression units relative to PAX3 levels in the RD ERMS cell line.

completion under a constant set of conditions, expression of these genes could be directly compared within and among samples. Of the 20 ARMS samples examined, we found that the PAX7 expression level was significantly less than the wild-type PAX3 expression level in 15 of 20 tumors and that the PAX3 and PAX7 mRNA levels were comparable in four of 20 tumors. Therefore, in the majority of ARMS tumors studied, wild-type PAX7 mRNA was expressed at lower levels than wild-type PAX3, and was often undetectable.

\section{Discussion}

In this study of downstream targets of the PAX3FKHR fusion transcription factor in the myogenic soft-tissue tumor ARMS, we complemented our series of long-term RD subclones constitutively expressing PAX3-FKHR by developing, characterizing, and implementing an inducible PAX3-FKHR cell culture system. By fusing a modified ER hormone-binding domain to the C-terminal end of the PAX3-FKHR open reading frame, we generated a transcription factor whose function is dependent on the presence of the ligand 4-OHT. In the absence of 4-OHT, several pieces of data indicated that this P3FK/ER protein has low but not negligible level of activity in RD cells. Following stimulation with the ligand 4-OHT, there is a progressive increase in activity with a greater than one order of magnitude change at the optimal dose. Since this expression change occurs with preformed protein, these experiments were also performed in the presence of the protein synthesis inhibitor CHX to assess whether candidate genes are direct targets of PAX3-FKHR.
Therefore, the combination of the inducible system and the stable subclones permits us to evaluate downstream expression events from both short-term and long-term perspectives.

In this study, we used these engineered cell culture resources to extend our understanding of a series of candidate target genes that were originally identified in gene expression studies of ARMS cell lines and tumors. The first such candidate gene is CXCR4 that encodes a G-protein coupled chemokine receptor bound by CXCL12/SDF-1. CXCR4/SDF-1 signaling induces directional motility, and has been hypothesized to be involved in the metastasis of multiple different types of cancers, including chronic lymphocytic leukemia, ${ }^{24}$ pancreatic carcinoma ${ }^{25}$ prostate carcinoma, ${ }^{26}$ multiple myeloma, ${ }^{27}$ astroglioma, ${ }^{28}$ and breast carcinoma. ${ }^{29}$ Additionally, our recent studies demonstrated that SDF-1 treatment of ARMS cells, which express relatively high levels of CXCR4, results in increased in vitro migration, adhesion, and matrix degradation. ${ }^{21}$ In addition, transfer of the PAX3-FKHR gene into ERMS cells is sufficient to make these cells responsive to several early events of CXCR4/SDF-1 signaling, and capable of directional chemotaxis towards a gradient of SDF-1.

An important question raised by these findings is whether the CXCR4/SDF-1 signaling pathway is responsible for the distinctive metastatic properties of ARMS. ARMS is an aggressive tumor with the propensity for early and wide metastatic dissemination, often involving bone marrow. ${ }^{30,31}$ Furthermore, within the ARMS category, the PAX3-FKHR-expressing tumors have a higher frequency of bone marrow metastasis than PAX7-FKHR-expressing tumors. ${ }^{32}$ The ERMS tumors as a group have a lower frequency 
of metastasis and are associated overall with a better outcome. ${ }^{31}$ Our RT-PCR expression data on the RMS tumors indicate that all RMS tumor types (PAX3FKHR- and PAX7-FKHR-expressing ARMS and fusion-negative ERMS) express comparable levels of CXCR4, and thus there is no evidence at the CXCR4 mRNA expression level for differences between tumor subsets. We have performed preliminary immunohistochemistry studies on ARMS and ERMS tumors, and have detected CXCR4 protein expression in tumors from each category without clear evidence of a difference in protein expression between the two RMS categories (data not shown). Based on the available data, we conclude that differential CXCR4 gene expression and presumably differential CXCR4/SDF-1 signaling is not responsible for the differential metastatic behavior between PAX3-FKHR and PAX7-FKHR ARMS subsets or between ARMS and ERMS categories. Nevertheless, these findings establish the transcriptional basis of a potentially important part of the metastatic machinery of the ARMS cell, and certainly require further studies to dissect the role of CXCR4/SDF-1 signaling in ARMS metastatic behavior. The availability of a CXCR4 monoclonal antibody $^{29}$ and small molecular inhibitor ${ }^{33}$ will facilitate these studies and comparison of these activities between various RMS subsets.

The second set of candidate target genes examined in this paper was wild-type $P A X 3$ and $P A X 7$. In our previous studies in ARMS tumors, we observed that PAX3-FKHR and PAX7-FKHR were expressed at higher levels than wild-type PAX3 and PAX7, respectively. ${ }^{4}$ The mechanism for PAX7FKHR overexpression involved at least in part in vivo amplification of the $P A X 7-F K H R$ fusion gene, whereas the mechanism for PAX3-FKHR overexpression was a copy number-independent increase in the $P A X 3-F K H R$ gene transcriptional rate. Since the wild-type proteins, which are transcriptionally less active than the fusion proteins $^{5,6}$ can potentially compete for binding sites with the fusion proteins, there is a biological impetus for overexpressing the fusion proteins. Based on the frequent occurrence of feedback regulation in genes involved in development, we reasoned that there may be mechanisms for downregulation of the wild-type $P A X 3$ or $P A X 7$ genes affecting the fusion gene products. The surprising result was that PAX3-FKHR upregulated wild-type PAX3 expression and downregulated wild-type PAX7 expression. The molecular mechanisms underlying these two regulatory events differed as evidenced by the finding of maximal downregulation of PAX7 in the inducible system without any added 4-OHT in contrast to the 4-OHTdependent increase in PAX3 expression.

The finding of upregulation of wild-type PAX3 by PAX3-FKHR suggests the possibility that there may be a pathway by which the $P A X 3-F K H R$ locus is upregulated by its gene product. The question then arises whether the levels of upregulation of the wildtype and fusion locus are comparable or whether changes in the structure of the fusion locus may permit this locus to respond with a higher level of gene expression. In this way, this autoregulation pathway may contribute to the phenomenon of PAX3-FKHR gene overexpression.

Our finding of PAX3-FKHR-induced downregulation of PAX7 expression is consistent with previous findings in both the cancer and developmental literature. The recent finding of PAX7 expression only in RMS cases without PAX3-FKHR or PAX7FKHR expression ${ }^{34}$ is similar to our findings in this study and in our past studies of RMS tumors ${ }^{23}$ and can now be explained by our finding of downregulation of PAX7 expression by the fusion transcription factor. In addition, this decreased PAX7 expression appears to mirror the developmental situation in which Pax3 is reported to repress Pax7 expression in the neural tube and somite regions. ${ }^{35}$ In normal murine embryos, Pax7 expression is restricted to medial neural tube and limited regions of the somite. In homozygous Splotch murine embryos (in which the Pax3 gene is mutated and inactivated), Pax7 gene expression is upregulated in the dorsal neural tube and regions of the somite where Pax3 is normally expressed. Therefore, in both ARMS tumorigenesis and normal development, there is evidence of natural controls to dampen PAX7 activity suggesting that PAX7 function may be detrimental and not redundant with PAX3.

In closing, these studies have highlighted the utility of the combination of the inducible P3FK/ER system and stable constitutively expressing PAX3FKHR subclones to study short-term and long-term gene expression changes. We have demonstrated several instances where these systems were used in a complementary manner to identify important genes whose expression is altered by PAX3-FKHR. We acknowledge that these studies of downstream target genes focused on the RNA expression level. However, demonstration of upregulation of CXCR4 protein expression in a representative PAX3-FKHRexpressing RD clone by FACS analysis has been presented previously. ${ }^{21}$ Furthermore, for PAX3 and PAX7 expression, the level of protein expression is too low to be detectable by Western analysis. Subsequent studies focusing on these downstream genes can move in one direction to focus on the promoter region and define the cis-acting elements responsible for this regulation, or can move in the opposite direction to further explore the functional and phenotypic consequences of the regulatory event. In the future, these cell culture systems will provide valuable tools to evaluate additional candidate genes and to identify larger sets of genes by expression array screens. As demonstrated above, many biological and clinical issues are raised as these downstream targets are identified that will stimulate further investigation with the goal of 
elaborating and analyzing the pathways that are disturbed during ARMS tumorigenesis and progression.

\section{Acknowledgements}

We thank Ms Lauren Nauta and Dr Richard Davis for their contributions to the early phases of this study. This study was supported in part by NIH Grants CA64202 and CA71838 (to FGB).

\section{References}

1 Barr FG. Gene fusions involving PAX and FOX family members in alveolar rhabdomyosarcoma. Oncogene 2001;20:5736-5746.

2 Davis RJ, D'Cruz CM, Lovell MA, et al. Fusion of PAX7 to FKHR by the variant $\mathrm{t}(1 ; 13)(\mathrm{p} 36 ; \mathrm{q} 14)$ translocation in alveolar rhabdomyosarcoma. Cancer Res 1994;54: 2869-2872.

3 Galili N, Davis RJ, Fredericks WJ, et al. Fusion of a fork head domain gene to PAX3 in the solid tumour alveolar rhabdomyosarcoma. Nat Genet 1993;5: 230-235.

4 Davis RJ, Barr FG. Fusion genes resulting from alternative chromosomal translocations are overexpressed by gene-specific mechanisms in alveolar rhabdomyosarcoma. Proc Natl Acad Sci USA 1997;94: 8047-8051.

5 Bennicelli JL, Edwards RH, Barr FG. Mechanism for transcriptional gain of function resulting from chromosomal translocation in alveolar rhabdomyosarcoma. Proc Natl Acad Sci USA 1996;93:5455-5459.

6 Bennicelli JL, Advani S, Schafer BW, et al. PAX3 and PAX7 exhibit conserved cis-acting transcription repression domains and utilize a common gain of function mechanism in alveolar rhabdomyosarcoma. Oncogene 1999;18:4348-4356.

7 del Peso L, Gonzalez VM, Hernandez R, et al. Regulation of the forkhead transcription factor FKHR, but not the PAX3- FKHR fusion protein, by the serine/ threonine kinase Akt. Oncogene 1999;18:7328-7333.

8 Lam PY, Sublett JE, Hollenbach AD, et al. The oncogenic potential of the Pax3-FKHR fusion protein requires the Pax3 homeodomain recognition helix but not the Pax3 paired-box DNA binding domain. Mol Cell Biol 1999;19:594-601.

9 Scheidler S, Fredericks WJ, Rauscher FJ, et al. The hybrid PAX3-FKHR fusion protein of alveolar rhabdomyosarcoma transforms fibroblasts in culture. Proc Natl Acad Sci USA 1996;93:9805-9809.

10 Ayyanathan K, Fredericks WJ, Berking C, et al. Hormone-dependent tumor regression in vivo by an inducible transcriptional repressor directed at the PAX3-FKHR oncogene. Cancer Res 2000;60: 5803-5814.

11 Bernasconi M, Remppis A, Fredericks WJ, et al. Induction of apoptosis in rhabdomyosarcoma cells through down-regulation of PAX proteins. Proc Natl Acad Sci USA 1996;93:13164-13169.

12 Epstein JA, Lam P, Jepeal L, et al. Pax3 inhibits myogenic differentiation of cultured myoblast cells. J Biol Chem 1995;270:11719-11722.
13 Khan J, Bittner ML, Saal LH, et al. cDNA microarrays detect activation of a myogenic transcription program by the PAX3-FKHR fusion oncogene. Proc Natl Acad Sci USA 1999;96:13264-13269.

14 Ginsberg JP, Davis RJ, Bennicelli JL, et al. Up-regulation of MET but not neural cell adhesion molecule expression by the PAX3-FKHR fusion protein in alveolar rhabdomyosarcoma. Cancer Res 1998;58: 3542-3546.

15 Bennicelli JL, Fredericks WJ, Wilson RB, et al. Wild type PAX3 protein and the PAX3-FKHR fusion protein of alveolar rhabdomyosarcoma contain potent, structurally distinct transcriptional activation domains. Oncogene 1995;11:119-130.

16 Bar FG, Chatten J, D’Cruz CM, et al. Molecular assays for chromosomal translocations in the diagnosis of pediatric soft tissue sarcomas. JAMA 1995;273: $553-557$.

17 Littlewood TD, Hancock DC, Danielian PS, et al. A modified oestrogen receptor ligand-binding domain as an improved switch for the regulation of heterologous proteins. Nucleic Acids Res 1995;23:1686-1690.

18 Breier G, Bucan M, Francke U, et al. Sequential expression of murine homeo box genes during F9 EC cell differentiation. EMBO J 1986;5:2209-2215.

19 Anderson J, Ramsay A, Gould S, et al. PAX3-FKHR induces morphological change and enhances cellular proliferation and invasion in rhabdomyosarcoma. Am J Pathol 2001;159:1089-1096.

20 Moore MA. The role of chemoattraction in cancer metastases. Bioessays 2001;23:674-676.

21 Libura J, Drukala J, Majka M, et al. CXCR4-SDF-1 signaling is active in rhabdomyosarcoma cells and regulates locomotion, chemotaxis, and adhesion. Blood 2002;100:2597-2606.

22 Hollenberg SM, Cheng PF, Weintraub H. Use of a conditional MyoD transcription factor in studies of MyoD trans-activation and muscle determination. Proc Natl Acad Sci USA 1993;90:8028-8032.

23 Barr FG, Fitzgerald JC, Ginsberg JP, et al. Predominant expression of alternative PAX3 and PAX7 forms in myogenic and neural tumor cell lines. Cancer Res 1999;59:5443-5448.

24 Burger JA, Burger M, Kipps TJ. Chronic lymphocytic leukemia B cells express functional CXCR4 chemokine receptors that mediate spontaneous migration beneath bone marrow stromal cells. Blood 1999;94:3658-3667.

25 Koshiba T, Hosotani R, Miyamoto Y, et al. Expression of stromal cell-derived factor 1 and CXCR4 ligand receptor system in pancreatic cancer: a possible role for tumor progression. Clin Cancer Res 2000;6: 3530-3535.

26 Taichman RS, Cooper C, Keller ET, et al. Use of the stromal cell-derived factor-1/CXCR4 pathway in prostate cancer metastasis to bone. Cancer Res 2002;62: 1832-1837.

27 Sanz-Rodriguez F, Hidalgo A, Teixido J. Chemokine stromal cell-derived factor-1alpha modulates VLA-4 integrin-mediated multiple myeloma cell adhesion to CS-1/fibronectin and VCAM-1. Blood 2001;97: 346-351.

28 Oh JW, Drabik K, Kutsch O, et al. CXC chemokine receptor 4 expression and function in human astroglioma cells. J Immunol 2001;166:2695-2704.

29 Muller A, Homey B, Soto $\mathrm{H}$, et al. Involvement of chemokine receptors in breast cancer metastasis. Nature 2001;410:50-56. 
30 Reid MM, Saunders PW, Bown N, et al. Alveolar rhabdomyosarcoma infiltrating bone marrow at presentation: the value to diagnosis of bone marrow trephine biopsy specimens. J Clin Pathol 1992;45: 759-762.

31 Tsokos M, Webber BL, Parham DM, et al. Rhabdomyosarcoma. A new classification scheme related to prognosis. Arch Pathol Lab Med 1992;116: 847-855.

32 Sorensen PH, Lynch JC, Qualman SJ, et al. PAX3-FKHR and PAX7-FKHR gene fusions are prognostic indicators in alveolar rhabdomyosarcoma: a report from the

children's oncology group. J Clin Oncol 2002;20: 2672-2679.

33 Tamamura H, Xu Y, Hattori T, et al. A low-molecularweight inhibitor against the chemokine receptor CXCR4: a strong anti-HIV peptide T140. Biochem Biophys Res Commun 1998;253:877-882.

34 Tiffin N, Williams RD, Shipley J, et al. PAX7 expression in embryonal rhabdomyosarcoma suggests an origin in muscle satellite cells. Br J Cancer 2003;89:327-332.

35 Borycki AG, Li J, Jin F, et al. Pax3 functions in cell survival and in pax7 regulation. Development 1999;126: 1665-1674. 